www.nature.com/cr

\title{
LKB1-AMPK axis revisited
}

\author{
Filippos Kottakis ${ }^{1}$, Nabeel Bardeesy ${ }^{1}$ \\ ${ }^{\prime}$ Massachusetts General Hospital Cancer Center, Harvard Medical School, Boston, MA 02114, USA \\ Cell Research (2012) 22:1617-1620. doi:10.1038/cr.2012.108; published online 17 July 2012
}

The LKB1 tumor suppressor encodes a serine-threonine kinase whose substrates control cell metabolism, polarity, and motility. LKB1 is a major mediator of the cellular response to energy stress via activation of the master regulator of energy homeostasis, AMPK. While mutational inactivation of $L K B 1$ promotes the development of many types of epithelial cancer, a recent report in Nature by Jeon et al. demonstrates that the LKB1-AMPK pathway can also have an unexpected positive role in tumorigenesis, acting to maintain metabolic homeostasis and attenuate oxidative stress thereby supporting the survival of cancer cells.

Normal mammalian cells possess adaptive mechanisms that enable coupling of nutrient availability with demand via integrated control of growth and metabolism. The widespread deregulation of these processes is now recognized as a prominent hallmark of all cancers. A key nutrient sensor in normal and cancer cells is the LKB1AMPK axis, which is critical for maintenance of metabolic homeostasis [1]. In response to energy stress (and resulting increase in AMP:ATP ratio), LKB1 phosphorylates AMPK, which in turn phosphorylates numerous substrates controlling diverse metabolic processes, with the net effect of shifting the balance

Correspondence: Nabeel Bardeesy

E-mail: bardeesy.nabeel@mgh.harvard.edu from anabolic to catabolic function and thereby restoring cellular ATP levels. LKB1 is an established tumor suppressor that is mutationally inactivated in a wide variety of epithelial cancers and promotes tumorigenesis when deleted in mouse models. While the underlying mechanisms for LKB1-mediated tumor suppression are not fully defined, the key role of AMPK in inactivating mTOR is thought to contribute to this process $[1,2]$.

An interesting paradox given this function as a tumor suppressor emerges from the observations that LKB1 or AMPK deletion renders primary cells resistant to transformation by overexpressed oncogenes and causes decreased viability of both cancer cell lines and primary cells under energy stress conditions [3-8]. The significance of the survival function of the LKB1-AMPK axis in cancer pathogenesis and the associated molecular mechanisms are the main focus of a recent report by Jeon et al. [9].

In this study, the authors utilized the A549 lung cancer cell line, which exhibits homozygous inactivating mutations of endogenous LKB1, as a model to study LKB1-AMPK-dependent survival under energy stress. Reintroduction of LKB1 resulted in the expected activation of AMPK and improved cell survival upon glucose deprivation. This effect was independent of mTOR or p53 inactivation, insofar as rapamycin treatment or p53 dominant-negative coexpression did not affect the starvation-induced cell death in A549 vector-transduced (i.e., control) cells.

Glucose starvation inhibits the pentose phosphate pathway (PPP), which is an important mechanism for NADPH production and consequent $\mathrm{H}_{2} \mathrm{O}_{2}$ detoxification (Figure 1). To survive in this setting, cells require compensatory NADPH generation, produced by other biochemical pathways. The authors hypothesized that a requirement for LKB1 in this adaptive NAPDH production may underlie its survival function in glucose-deprived cells. Consistent with this hypothesis, they showed that treatment with $\mathrm{N}$-acetylcysteine or catalase, both antioxidants, inhibited starvation-induced death of both LKB1- and AMPK-deficient (A549/ HeLa and MEFs, respectively) cells. In addition, metabolic analysis of the glucose-starved A549 cells revealed that the ratios of NADP/NADPH and oxidized glutathione/reduced glutathione (GSSG/GSH) were maintained in LKB1-transduced cells, whereas both ratios were increased in the vector-transduced cells. Since NAPDH is mainly utilized to reduce GSSG to its GSH form, which is in turn used to detoxify cells from $\mathrm{H}_{2} \mathrm{O}_{2}$ through the function of glutathione peroxidase, these results reveal that the LKB1-AMPK axis has a central role in suppressing oxidative stress (Figure 1).

Upon glucose starvation and consequent loss of PPP function, the major contributor to NADPH generation is mitochondrial metabolism whose activity 
is maintained by fatty acid oxidation in this context. The rate-limiting enzyme in catabolism of fatty acids is carnitine palmitoyltransferase 1 (CPT1). Under normal conditions, CPT1 is inhibited by the malonyl-CoA produced by acetylCoA carboxylase alpha (ACC1) and acetyl-CoA carboxylase beta (ACC2). These two enzymes are subject to inhibition by phosphorylation by AMPK [10]. Therefore, the authors hypothesized that LKB1-AMPK may control the levels of NADPH by inhibiting ACC 1 and ACC2. Targeted knockdown studies revealed that $\mathrm{ACC} 2$ inactivation was sufficient to restore NADP/NADPH and GSSG/GSH ratios and to rescue cell death in glucose-starved A549 cells. These findings were extended by a set of experiments using the constitutively active ACC2 (S212A) mutant, the fatty acid synthase (FAS) inhibitor $\mathrm{C} 75$, the ACC inhibitor TOFA, malate supplement, buthionine sulphoximine (which depletes GSH), and nicotinamide, that together support the hypothesis that survival under glucose starvation is dependent on the inactivation of $\mathrm{ACC} 2$ (and ACC1 in some cell types) by AMPK-regulated phosphorylation.

Matrix detachment impairs cell viability in part due to induction of energy stress, leading to NADPH depletion and increased $\mathrm{H}_{2} \mathrm{O}_{2}$ levels. The authors found that the LKB1-AMPK axis also plays a pro-survival role in this setting. LKB1-null cells have reduced viability and impaired growth under anchorageindependent conditions, due mainly to decreased NADPH levels and subsequent oxidative stress.

Cancer cells need to activate survival mechanisms to cope with energy stress and matrix detachment during tumor progression. Thus, the authors speculated that the LKB1-AMPKACC1/2-NADPH pathway might play an important role in promoting tumor growth. Correspondingly, NAC treatment or shRNA-mediated knockdown of ACC $1 / 2$ increased anchorage-independent growth of A549 cells in soft agar, and ACC1/2 knockdown enhanced tumorigenicity in xenograft studies. Moreover, similar effects were seen using RAS V12-transformed AMPK $\alpha^{-1-}$ MEF cells with concurrent ACC $1 / 2$ knockdown, consistent with LKB1 and AMPK acting in a common pathway to promote tumorigenesis.

The results described above were obtained mainly in the setting of ectopically restoring LKB1 in LKB1-deficient cancer cells. An important question that is raised by these findings is whether cancers that arise with an intact LKB1AMPK axis require this pathway for sustained tumorigenesis. To address this issue, the authors examined the impact of knockdown of either LKB1 or AMPK $\alpha 1$ in MCF7 breast cancer cells. These manipulations reduced xenograft tumor formation, as did overexpression of the constitutively active, phosphorylation-deficient mutants ACC mutant (ACC1-S79A or ACC2-S212A). Collectively, these observations indicate that activation of AMPK and consequent inactivation of $\mathrm{ACC} 1 / 2$ by endogenous AMPK is an important survival mechanism in cancer (Figure 1).

These conclusions are of particular

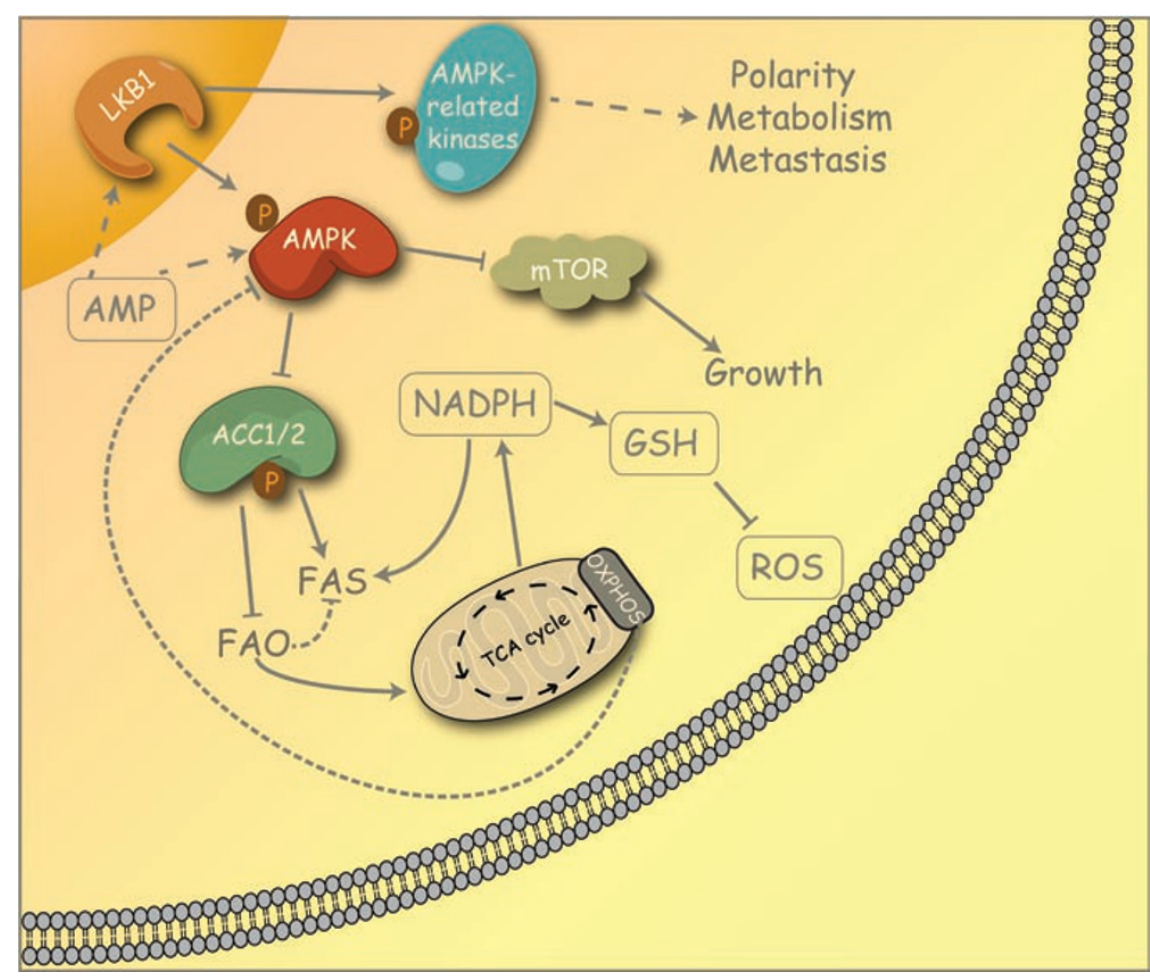

Figure 1 AMPK is phosphorylated and activated by LKB1 in response to an increasing cellular AMP:ATP ratio (which reflects a decrease in energy supply). AMPK in turn phosphorylates and inactivates ACC1/2, promoting a shift from fatty acid synthesis (FAS) to fatty acid oxidation (FAO). FAS depletes NADPH that is required for $\mathrm{H}_{2} \mathrm{O}_{2}$ detoxification. $\mathrm{FAO}$, by contrast, produces metabolites that are used by the TCA cycle, resulting in increased NADPH and enhanced cell survival. This pathway may only be transiently activated in glucose-deprived cells since ATP, produced by the coupling the TCA cycle with oxidative phosphorylation (OXPHOS), will eventually inhibit AMPK. In addition to the role of the LKB1-AMPK pathway in facilitating tumor cell survival, LKB1 is a context-specific tumor suppressor, which acts to control cell polarity and restrict cell growth via mTOR inactivation and induction of other AMPK-related kinases. 
note considering the established role of LKB1 in tumor suppression. This tumor suppressive function may involve the ability of the LKB1-AMPK pathway to promote $\mathrm{mTOR}$ inactivation by TSC2 and raptor phosphorylation $[1,2]$, as well as functions of other members of the family of AMPK-related kinases. Since mTOR activation is a common feature of cancer and a driver of many tumor types, it may seem counterintuitive to assign a tumorigenic role in one of its major inhibitors. Another layer of complexity stems from the fact that AMPK activation is unlikely to be sustained for prolonged period of time. Fatty acid oxidation, activated by AMPK during glucose starvation, will feed into the TCA-oxidative phosphorylation cycles, in turn leading to ATP production and AMP:ATP ratio decrease, followed by AMPK inactivation (Figure 1). To reconcile these two opposing functions of AMPK, the authors suggest that this negative feedback loop is a reflection of the temporal manner of AMPK activation that, at physiological levels, is essential for the survival of the tumor cells during energy stress (starvation or matrix detachment) but it is quickly followed by inactivation. According to this model, use of LKB1 or AMPK inhibitors in acute regimens could prove beneficial for cancer therapy by sensitizing cells to energy stress. Moreover, the acute nature of the treatment could potentially cause metabolic stress, sensitizing cells to other chemotherapy regiments. Sustained inactivation of the LKB1-AMPK pathway on the other hand, could result in long-term stress, promote rewiring of intracellular metabolic processes, and tip the balance towards increased proliferation due to activation of mTOR and other pathways.

Based on these results, a major question is raised: How do LKB1-deficient tumors bypass the normal requirement for the LKB1-AMPK axis in energy stress response? Presumably, LKB1 inactivation must occur in the context of specific cooperating molecular alterations that enable cell survival despite these impairments in metabolic homeostasis, thereby allowing the pro-tumorigenic consequences of LKB1 loss to take hold. One potential escape route could involve alternative, LKB1-independent mechanisms for AMPK activation such as induction of CAMK2 or a hexokinase-dependent pathway [1], but other pathways could be equally important. Further studies will likely uncover additional adaptive processes permitting cell survival under metabolic stress in the absence of LKB1. Metabolic profiling of LKB1 null tumors could provide a glimpse of these alternative pathways, opening the way for new targeted therapeutic strategies. Moreover, cancer genome sequencing efforts are likely to reveal specific complementation groups of mutations that coexist with LKB1 mutations in different cancer types or are mutually exclusive, reflecting molecular pathways that synergize with LKB1 deficiency. Additionally, other important AMPK-independent functions of LKB1 should also be brought into focus. In this regard, AMPK $\alpha 1 / 2$ are constituents of a 14-member family of kinases that are phosphorylated and activated by LKB1 and that broadly include regulators of epithelial cell polarity as well as metabolism. Disruption of polarity, as altered metabolism, is a hallmark of epithelial cancer progression [11], and the relative roles of these processes downstream of LKB1 in growth control is an area of active investigation. Indeed, inactivation of LKB1 produces dramatic invasive and migratory phenotypes in different cancer models [12-14]. The findings of Jeon et al. [9] may thus presage that the LKB1-AMPK axis is only a minor component of the LKB1 tumor suppressor program compared to its functions in metabolic adaptation and cell survival.

Long-term use of metformin, in the treatment of Type II diabetes, has been shown to reduce tumor incidence and sensitizes multiple cancer cell types to chemotherapy [1]. Furthermore, LKB1 controls hepatic glucose metabolism and the therapeutic effects of metformin. A recent study that revealed that AMPK is activated by salicylate also suggests that this mechanism is, at least partly, responsible for the cancerprotective effects of aspirin [15]. On the other hand, inhibition of LKB1-AMPK sensitizes cancer cells to energy stressinduced apoptosis. Therefore, the results presented by Jeon et al. [9] suggest that targeting the LKB1-AMPK axis in cancer should be done with caution and with attention to specific contexts.

\section{References}

1 Shackelford DB, Shaw RJ. The LKB1AMPK pathway: metabolism and growth control in tumour suppression. Nat Rev Cancer 2009; 9:563-575.

2 Contreras CM, Akbay EA, Gallardo $\mathrm{TD}$, et al. Lkb1 inactivation is sufficient to drive endometrial cancers that are aggressive yet highly responsive to mTOR inhibitor monotherapy. Dis Model Mech 2010; 3:181-193.

3 Bardeesy N, Sinha M, Hezel AF, et al. Loss of the Lkb1 tumour suppressor provokes intestinal polyposis but resistance to transformation. Nature 2002; 419:162-167.

4 Kato K, Ogura T, Kishimoto A, et al. Critical roles of AMP-activated protein kinase in constitutive tolerance of cancer cells to nutrient deprivation and tumor formation. Oncogene 2002; 21:6082-6090.

5 Laderoute KR, Amin K, Calaoagan JM, et al. 5'-AMP-activated protein kinase (AMPK) is induced by low-oxygen and glucose deprivation conditions found in solid-tumor microenvironments. $\mathrm{Mol}$ Cell Biol 2006; 26:5336-5347.

6 Chhipa RR, Wu Y, Mohler JL, Ip C. Survival advantage of AMPK activation to androgen-independent prostate cancer cells during energy stress. Cell Signal 2010; 22:1554-1561.

7 Shaw RJ, Kosmatka M, Bardeesy N, et al. The tumor suppressor LKB1 kinase directly activates AMP-activated kinase and regulates apoptosis in response to energy stress. Proc Natl Acad Sci USA 2004; 101:3329-3335.

8 Carretero J, Medina PP, Blanco R, et 
al. Dysfunctional AMPK activity, signalling through mTOR and survival in response to energetic stress in LKB1deficient lung cancer. Oncogene 2007; 26:1616-1625.

9 Jeon SM, Chandel NS, Hay N. AMPK regulates NADPH homeostasis to promote tumour cell survival during energy stress. Nature 2012; 485:661-665.

10 Hardie DG, Pan DA. Regulation of fatty acid synthesis and oxidation by the AMP-activated protein kinase. Bio- chem Soc Trans 2002; 30:1064-1070.

11 Martin-Belmonte F, Perez-Moreno M. Epithelial cell polarity, stem cells and cancer. Nat Rev Cancer 2012; 12:2338.

12 Ji H, Ramsey MR, Hayes DN, et al. LKB1 modulates lung cancer differentiation and metastasis. Nature 2007; 448:807-810.

13 Liu W, Monahan KB, Pfefferle AD, et al. LKB1/STK11 inactivation leads to expansion of a prometastatic tumor subpopulation in melanoma. Cancer Cell 2012; 21:751-764.

14 Cheng H, Liu P, Wang ZC, et al. SIK1 couples LKB1 to p53-dependent anoikis and suppresses metastasis. Sci Signal 2009; 2:ra35.

15 Hawley SA, Fullerton MD, Ross FA, et $a l$. The ancient drug salicylate directly activates AMP-activated protein kinase. Science 2012; 336:918-922. 